\title{
Elektrophoretische Trennung der Serumlipoproteine in Agarosegel mit Albuminzusatz
}

\author{
Von N. Zöllner, W. Gröbner, Christa Berger und G. Wolfram \\ Aus der Medizinischen Poliklinik der Universität Müncben (Direktor: Prof. Dr. W. Seitz) \\ (Eingegangen am 9. Juni 1969)
}

Die Elektrophorese der Serumlipoproteine in Agarosegel (0,8\%) mit Albuminzusatz (0,26\%) liefert eine scharfe Trennung in gleichmäßige Banden von Chylomikronen, $\beta$-Lipoproteinen, prä- $\beta$-Lipoproteinen und zwei $\alpha$-Lipoproteinen. Bei einzelnen Hyperlipidämien finden sich zusätzliche Banden im Bereich $\operatorname{der} \beta$ - und prä- $\beta$-Lipoproteine.

Die Bedingungen der elektrophoretischen Trennung (Konzentration des Agarosegels und des Albumins, Puffer und Trennstrecke), das Trocknen der Schicht, die Färbung der Banden und die densitometrische Auswertung werden näher untersucht.

Die Lokalisierung der Fraktionen erfolgt durch Fett- und Proteinfärbung; durch Herstellung einer Abklatschkopie auf Chromatographiepapier können die Färbungen an der gleichen Trennung vorgenommen werden. Durch präparative Trennung in Agarosegel oder Fällung $\operatorname{der} \beta$-Lipoproteine und Elektrophorese nach Wiederauflösung können einzelne Fraktionen isoliert und die Eiweißkomponenten immunologisch nachgewiesen werden.

\section{Electrophoretic separation of serum lipoproteins in agarose gel with the addition of albumin}

Electrophoresis of serum-lipoproteins in agarose $(0.8 \%)$ with added albumin $(0.26 \%)$ yields a good separation of uniform bands of chylomicrons, $\beta$-lipoproteins, pre- $\beta$-lipoproteins and of two fractions of $\alpha$-lipoproteins. In some hyperlipidaemias additional fractions in the zone of $\beta$ - and pre- $\beta$-lipoproteins can be demonstrated.

The factors infuencing the electrophoretic separation (i. e. concentration of agarose and albumin, buffer and migration distance), and the drying of the layer, the staining and the densitometric evaluation were studied.

The fractions are localized by staining for lipids or proteins. If both stains are required, e. g. for a comparison of bands, the agarose surface can be printed on to chromatography paper which by diffusion takes up sufficient material to permit protein staining. Fractions separated by electrophoresis can be extracted from the gel, thus permitting their isolation. Precipitation of $\beta$-lipoproteins also yields a clear-cut separation of $\alpha$ - and $\beta$-lipoprotein. Isolated fractions may be studied immunologically.

Im Jahre 1952 zeigte MAURER (1) mittels ${ }^{35} \mathrm{~S}$-markierten Serumproteinen und ${ }^{32} \mathrm{P}$-markiertem Phosphat, daß die papierelektrophoretische Verteilung von Lipidphosphor im Serum von Ratten und Kaninchen durch den $\mathrm{Zu}-$ satz von unmarkiertem Serum zum Puffer stark beeinflußt werden kann: Während unter den üblichen Bedingungen der Papierelektrophorese fast der gesamte Lipidphosphor in der Nähe des Auftragortes verblieb (Kaninchen) oder über den gesamten Wanderungsbereich verteilt war (Ratte), wurde nach Elektrophorese mit Serumzusatz zum Puffer eine deutliche Bande von ${ }^{32} \mathrm{P}$ (eluierbar mit Methanol-Eisessig) in der Gegend der $\alpha$-Globuline gefunden. Damit war eine entscheidende Voraussetzung für eine verbesserte Fraktionierung der bis dahin papierelektrophoretisch kaum zu trennenden Lipoproteine geschaffen. Es gelang aber erst LEES und Hatch (2) eine klinisch anwendbare Methode der Papierelektrophorese mit Albuminzusatz zu erarbeiten. Mit deren Hilfe konnten dann Fredrickson und LEES $(3,4)$ eine Verbesserung der Einteilung der Hyperlipidämien (Hyperlipoproteinämien) vorschlagen.

Die Ergebnisse der Lipoproteinelektrophorese auf Papier waren auch nach der erwähnten Verbesserung noch schwer reproduzierbar, auch war die Trennung einzelner Fraktionen, speziell der prä- $\beta$-Lipoproteine von $\operatorname{den} \beta$-Lipoproteinen, ungenügend. Eine wesentliche Verbesserung ergab sich erst durch die Einführung der Agarose als Trägermaterial. Thre Anwendung in der
Lipoproteinelektrophorese $(5,6)$ führte zu einer guten Abtrennung der prä- $\beta$-Fraktion; außerdem beschrieben die Autoren bis zu neun Lipoproteinfraktionen verschiedener Mobilitäten und verschiedener relativer Konzentrationen. Die Form der erzielten Banden legte nahe, auch bei der Lipoproteinelektrophorese in Agarose den Einfluß von Albumin zu untersuchen. Sowohl Hatch und Lees (7) als auch Noble (8) fanden, daß dies die Ungleichmäßigkeiten der Banden, speziell $\operatorname{der} \beta$ Lipoproteine, verhütet. Systematische Untersuchungen über die hierfür notwendigen Albuminkonzentrationen wurden jedoch nicht mitgeteilt.

Unabhängig von den genannten Autoren haben wir die gleiche Feststellung getroffen. Wir haben weitere Untersuchungen über die Verbesserung der Banden durch Albumin durchgeführt und eine Abklatschmethode zur Lokalisation der Proteine entwickelt; unter den von uns angewendeten Trennbedingungen kommt es darüber hinaus regelmäßig zu einer deutlichen Aufteilung der $\alpha$-Lipoproteine in zwei Fraktionen.

\section{Methodik}

Serum

Blut wird den Patienten 12 Stdn. nach der letzten Nahrungsaufnahme entnommen, 30 Min. bei Raumtemperatur aufbewahrt und dann zentrifugiert. Das Serum wird in die Lipoproteinelektrophorese eingesetzt. Aufbewahren von Serumproben bei $+4^{\circ}$ führt rasch zu Veränderungen der Lipoproteinmuster. 


\section{Lipoproteinelektrophorese}

Mit Veronal-Natriumphosphat vermischtes Agarosepulver (Instant-Gel A 08-82, Serva-Labor, Heidelberg) wird in $100 \mathrm{ml}$ dest. Wasser (Konzentration der Agarose 0,8\%, des Puffers 0,05M, $\mathrm{pH} 8,2)$ kurz aufgekocht. $\mathrm{Zu} 50 \mathrm{~m} /$ des etwa $40^{\circ}$ warmen, flüssigen Gels wird im Standardverfahren $1 \mathrm{~m} l$ einer mit $1 \mathrm{M}$ Trispuffer auf pH 8,2 eingestellten 13,3 proz. Rinderalbuminlösung (Behringwerke, Marburg) zugesetzt und gut durchmischt.

In genau horizontal gelagerten Trennkammern aus Kunststoff (Desaga, Heidelberg) werden $9 \times 12 \mathrm{~cm}$ große Glasplatten mit $50 \mathrm{~m} l$ des fertigen Gels übergossen, so da $\beta$ die mit Klebestreifen verschlossenen Verbindungsschlitze der Trennkammern auch ausgefüllt sind. Bei Raumtemperatur ist die etwa $3 \mathrm{~mm}$ dicke Schicht nach $30 \mathrm{Min}$. ausreichend fest.

Mit Schablone und Querstanze werden $3 \mathrm{~cm}$ von der Schmalseite der Platte entfernt im Abstand von $0,5 \mathrm{~cm}$ vier Vertiefungen $(2 \times 12 \mathrm{~mm})$ ausgehoben. Am Rand der Platte wird mit einer Rundstanze ein Loch $(\varnothing 2 \mathrm{~mm})$ für bromphenolblaumarkiertes Albumin angebracht, durch dessen Position der Verlauf der Trennung verfolgt werden kann.

In die vorgestanzten Vertiefungen werden $0,04 \mathrm{ml}$ eines Gemisches aus $0,2 \mathrm{~m} l$ Serum, $0,3 \mathrm{~m} l 0,9$ proz. $\mathrm{NaCl}$-Lösung und $0,5 \mathrm{~m} l$ Agarose-Gel pipettiert. Bei normalem Lipidgehalt des Serums können auch $0,04 \mathrm{ml}$ Serum direkt aufgetragen werden. Die Vertiefungen deckt man mit einem Tropfen flüssigen Gels ab.

Die Trennkammern werden nach Entfernen der Klebestreifen auf den mit Wasser gekühlten Block der UGI-Apparatur (Desaga, Heidelberg) gesetzt. Als Puffer dient 0,05M Veronal-Natriumphosphat-Puffer pH 8,2 (Serva-Labor, Heidelberg). Uber die mit Gel gefüllten Verbindungsschlitze der Trennkammer ist die Verbindung zwischen Gelschicht und Puffer gesichert. Die Trennung erfolgt in $90-110 \mathrm{Min}$. bei einer Netzspannung von $180 \mathrm{~V}$ (Netzgerät, Typ II, Desaga, Heidelberg) und einer Trennstrecke von $6 \mathrm{~cm}$, bezogen auf Albumin. Anschließend wird die Gelschicht in der Trennkammer mit etwa $20 \mathrm{~m} l$ 2proz. Essigsäurelösung übergossen und 30 Min. fixiert. Nach Abgießen der Essigsäurelösung werden die Platten aus der Trennkammer gehoben, mit feuchtem Chromatographiepapier (Schleicher \& Schüll Nr. 2043a) abgedeckt und zwei Stunden unter einem Kaltluftventilator getrocknet. Soll zu Zwecken des Vergleiches der Beweglichkeiten von Proteinen und Lipoproteinen, bzw. zur elektrophoretischen Zuordnung ein Abklatsch gemacht werden (s. unten), so muß dies vor der Fixierung erfolgen.

\section{Färbung}

Die Lipide werden mit Sudanschwarz B in Anlehnung an NoBLE (8) gefärbt. $400 \mathrm{mg}$ Sudanschwarz B werden in $200 \mathrm{ml} 60$ proz. Äthanol gelöst (Magnetrührer, eine Stunde). Nicht gelöste Substanz läßt man über Nacht sedimentieren. Nach Dekantieren verdünnt man die Stammlösung mit 60proz. Äthanol 1:2 ( $/ / \mathrm{v})$. In diese von einem Magnetrührwerk umgewälzte Lösung werden die getrockneten Platten mit der Schicht nach oben 2 Stdn. eingehängt. Die vom Lipoprotein freien Teile der Schicht werden anschließend durch vorsichtige Schaukelbewegungen der Platte in 60 proz. Äthanol in 20 Sek. wieder entfärbt. An der Luft ist die Agarosegel-Schicht schnell trocken und fertig für die weitere Auswertung.

Die Proteine auf den Agaroseplatten werden mit Amidoschwarz 10 B 30 Min. lang gefärbt (9). Die Farblösung besteht aus $1 \mathrm{~g}$ Amidoschwarz $10 \mathrm{~B}$ in $1 /$ eines Gemisches von $850 \mathrm{ml}$ Acetatpuffer und $150 \mathrm{~m} l$ Glycerin. Der Acetatpuffer wird aus $500 \mathrm{~m} l$ Essigsäure $(60 \mathrm{~m} /$ Eisessig auf $1 /$ dest. Wasser) und $500 \mathrm{~m} / \mathrm{Na}$ triumacetat-Lösung hergestellt. (13,6 g Natriumacetat-3-Hydrat auf $1 /$ dest. Wasser.) Die Agarosegelschicht läßt sich mit 2 proz. Essigsäure unter mehrmaligem Wechseln der Lösung' entfärben.

\section{Auswertung}

Zur Dokumentation eignet sich ein Negativabzug auf Photopapier oder Röntgenfilm. Die densitometrische Auswertung wird in einem Zusatzgerät zum Spektralphotometer DU G 4700 der Fa. Beckman Instruments bei $\lambda=575 \mathrm{~nm}$ durchgeführt (10) (siehe Abb. 1).

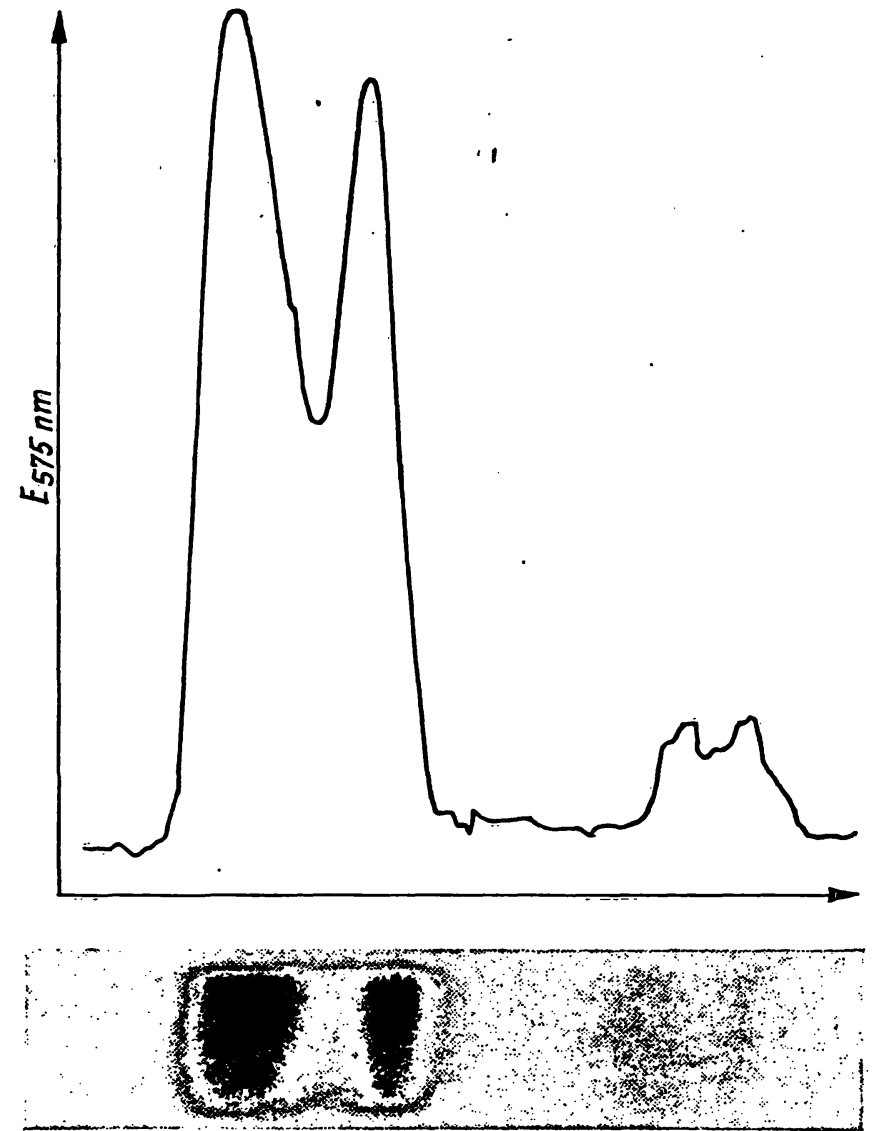

Abb. 1

Lipoproteinelektrophorese in Agarosegel mit Albuminzusatz (Standardmethode) und die entsprechende Densitometerkurve. Die Lipoproteine bewegen sich vom Auftragsort (links im Bild) in Richtung auf die Anode. Das Serum enthält keine Chylomikronen, die am Auftrags-
ort liegen blieben, aber $\beta-$, prä- $\beta-$ und $z w e i$-Lipoproteinfraktionen

\section{Ergebnisse}

\section{Versucbe zur Methode}

Bei Versuchen mit verschiedenen Albuminkonzentrationen im Agarosegel ergeben 0,266\% Albumin im Gel (Standardmethode) sehr gute Trennungen (Abb. 2). Schon bei $0,05 \%$ Albumin in der Schicht verschwindet die Sichelform der $\beta$-Lipoproteinbande, die schärfsten Banden erzielt man jedoch mit 0,21-0,79\% Albumin. Höhere Konzentrationen des Albumins führen $\mathrm{zu}$ keiner besseren Trennung, lassen aber beim Trocknen

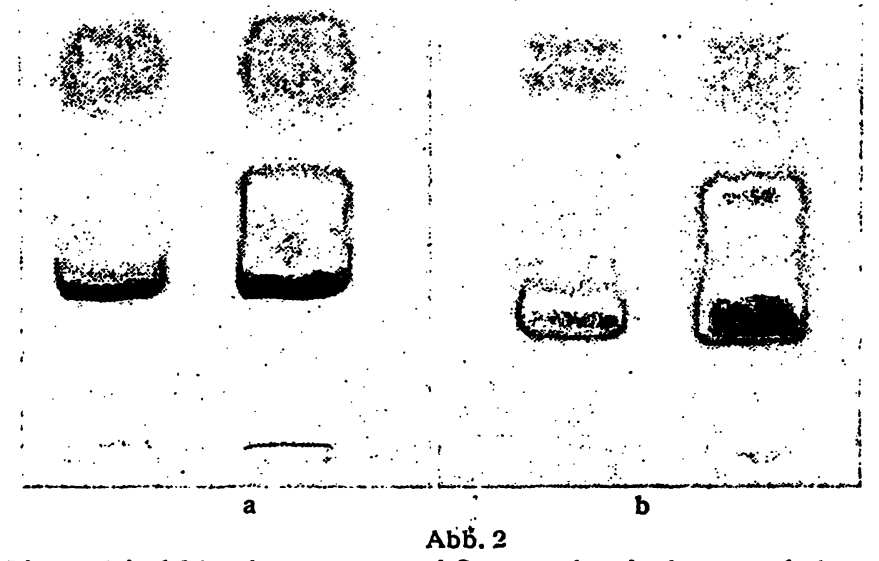

Lipoproteinelektrophorese von zwei Serumproben in Agarosegel ohne Albuminzusatz nach RAPP und KAHLKE (6) (a) und mit 0,266\% Albumin im Agarosegel (b, Standardmethode) 


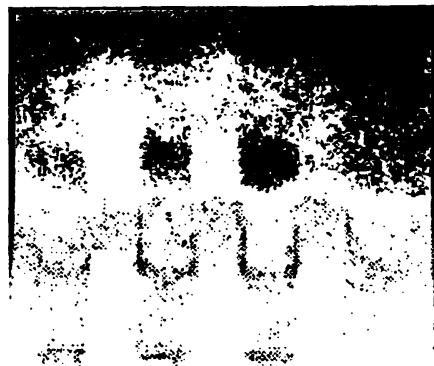

Abb. 3

Lipoproteinelektrophorese in Agarosegel mit Albuminzusatz (Standardmethode). Die Färbung mit Amidoschwarz (a) zeigt, daß das dem Agarosegel zugesetzte Albumin bis zur halben Länge der Platte wandert. Zum Vergleich (b) ist eine unter gleichen Bedingungen getrennte Platte, die mit Sudanschwarz B gefärbt wurde, gegenübergestell

das Filterpapier am Gel festkleben. Von Änderungen der Albuminkonzentrationen im Gel bleiben die Relationen der Lipoproteinkonzentrationen unbeeinflußt.

Das Albumin im Agarosegel wandert im elektrischen Feld bis etwa zur Hälfte der Plattenlänge anodenwärts (Abb. 3). Die Grenze der Albuminzone deckt sich mit keiner der Lipoproteinfraktionen, es liegt also kein Schleppeffekt vor. Obwohl die $\beta$-Lipoproteine außerhalb des Albumins aufgefunden werden, zeigt ihre Bande nicht die Unregelmäßigkeiten, die man von der Elektrophorese ohne Albuminzusatz kennt. Trägt man Serum im mittleren Drittel der Platte auf, so daß die Grenze des zugesetzten Albumins den Auftragspunkt nicht erreicht, erhält man die gleichen regelmäßigen Banden und den gleichen Trenneffekt, wie wenn das Albumin aus $\operatorname{dem} \beta$-Lipoprotein herauswandert.

Als Puffer wurden Veronal- bzw. Veronal-Natriumphosphat-Puffer (8) eingesetzt. Beide Puffer ergaben vergleichbar gute Trennungen. Da Veronal-Natriumphosphat-Puffer standardisiert im Handel ist, wird ihm der Vorzug gegeben.

Die günstigste Trennstrecke, liegt bei $6 \mathrm{~cm}$, da hier der Abstand $z$ wischen $\beta$ - und prä- $\beta$-Bande $1,1 \mathrm{~cm}$ beträgt. Auch kürzere Trennstrecken ergeben scharfe Trennungen, doch sind die Abstände zwischen den Fraktionen für die weitere Auswertung (Densitometrie, Elution) eventuell zu gering.

Zum Trocknen wird die Agarosegelschicht mit feuchtem Chromatographiepapier abgedeckt. Filterpapier eignet sich weniger gut, da Cellulosefasern an der Gelschicht haften. Beim Trocknen verliert die Agarosegelschicht etwa 98\% ihres Gewichtes. Lufttrocknen ohne Abdecken mit Papier läßt die Schicht reißen.

Mit der beschriebenen Technik ist eine Färbezeit von 2 Stdn. ausreichend. Eine längere Einwirkung der Farblösung bringt keine besseren Ergebnisse. Dagegen sind ähnlich gute Färbungen ohne Rühren erst nach 8 Stdn. zu erwarten.

Die Zeit des Entfärbens nach Sudanschwarz B kann bis auf 60 Sek. ausgedehnt werden, ohne daB die Farbintensität der Lipoproteinfraktionen abnimmt. Bei gleichmäßigem Bewegen der Platte im Entfärber kann man schon nach 20 Sek. ein entfärbtes Agarosegel erzielen.
Zur densitometrischen Auswertung wurde ein 0,5 mm breiter Spalt verwendet, der mit einer Länge von $11 \mathrm{~mm}$ die Banden gerade erfaßt. Da zwischen der $\beta$ - und der prä- $\beta$-Bande immer färbbares Material zu liegen kommt, kann keine vollständige Auflösung dieser beiden Fraktionen erzielt werden. Bei steigenden Serumeinsätzen bleiben aber die Relationen der Fraktionen zueinander gewahrt. Deshalb sind die Lipoproteinelektrophoresestreifen zur quantitativen Auswertung der Lipid- und Proteinanteile geeignet.

\section{Charakterisierung der Fraktionen}

Durch eine Fettfärbung werden auf den Agaroseplatten nur die Lipide der Lipoproteine erfaßt. Zur Charakterisierung der Lipoproteinmoleküle ist jedoch auch die Beschreibung des Proteinanteils unerläBlich.

Zum Vergleich der Wanderungsgeschwindigkeit mit der der großen Serumproteinfraktionen ist der übliche Weg die verschiedene Färbung von unter möglichst gleichen Bedingungen getrennten verschiedenen Proben. Einfacher ist ein Proteinabklatsch der Agarosegelplatte auf Papier, da so die nach spezifischen Färbungen sichtbaren Fett- und Eiweißanteile des gleichen Trennganges miteinander verglichen werden können.

Bei diesem Verfahren wird nach Abschluß der Lipoproteinelektrophorese (Einsatz 0,04 $\mathrm{ml}$ unverdünntes Serum) auf die feuchte Agarosegelschicht trockenes Chromatographiepapier (Schleicher und Schüll Nr. 2043a) gelegt und in einer feuchten Kammer bei $+4^{\circ}$ aufbewahrt. Nach einer Stunde wird der Papierstreifen vorsichtig abgezogen und die durch Diffusion eingedrungenen Proteine werden mit Amidoschwarz 10B in üblicher Weise angefärbt. Der Proteinabzug auf Papier kann auch densitometrisch ausgewertet werden. Ein Nachweis von Lipiden auf dem Papier gelingt nicht. In der Agarosegelschicht lassen sich die Lipide anschließend wie üblich mit Sudanschwarz färben, ohne $\mathrm{da} ß$ eine wesentliche Abschwächung, Verbreiterung oder Verzerrung der Banden festzustellen ist.

Der immunologische Nachweis von $\alpha$ - und $\beta$-Lipoproteinen wird nach der Trennung der Lipoproteine auf der gleichen Platte wie bei der Immunelektrophorese nach GrABAR (9) geführt.

Zum Ausschluß von Artefakten im Bereich $\operatorname{der} \beta$-Lipoproteine kann diese Fraktion isoliert und erneut in die Elektrophorese eingesetzt werden. Dabei dient das gleiche Serum als Referenzsubstanz. Die präparative Trennung geht von $0,2 \mathrm{ml}$ Serum aus, die unverdünnt in das vergtößerte Auftragsteservoir pipettiert werden. Anhand einer Referenzbahn, die abgetrennt und gefärbt wird, können die Lipoproteinbanden lokalisiert und mit der Schicht abgehoben werden. Der in der Ultrazentrifuge (10 Min. $20.000 \mathrm{~g}$ ) isolierte Überstand mit den $\beta$ Lipoproteinen $(\sim 1 \mathrm{ml})$ wird durch Dialyse gegen 6proz. Macrodex ${ }^{1}$ ) eingeengt. Davon werden $0,04 \mathrm{ml}$ mit dem ursprünglichen Serum als Referenzsubstanz

1) Knoll AG, Ludwigshafen. 


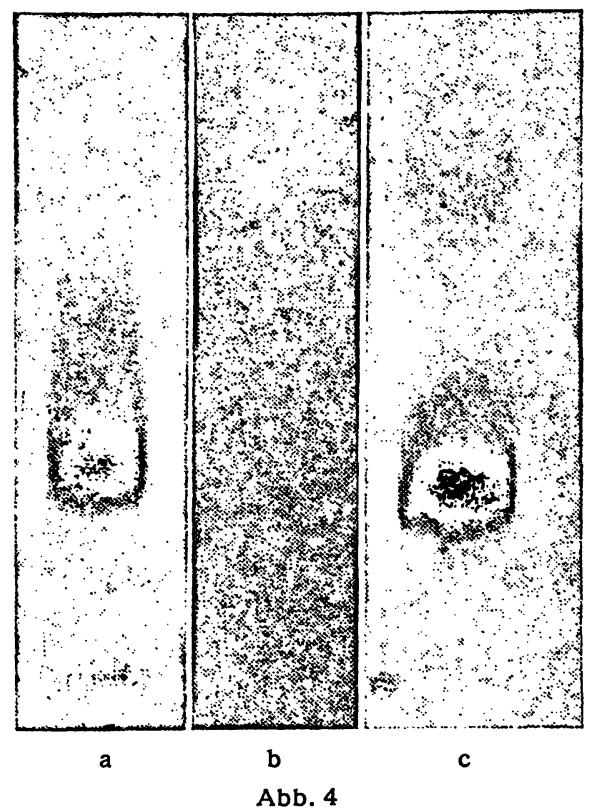

Elektrophorese :der nach BuRSTEIN (11) gefällten $\beta$-L.ipoproteine (a), der $\alpha$-Lipoproteine im Überstand (b) und der Lipoproteine im ursprünglichen Serum (c)

erneut in die Lipoproteinelektrophorese eingesetzt. Durch Fettfärbung und Präzipitation mit Anti- $\beta$-lipoprotein kann gezeigt werden, daß die Beweglichkeit der $\beta$-Lipoproteine nicht von der Anwesenheit der anderen Serumproteine abhängig ist und durch die Isolierung nicht beeinflußt wird.

Mittels Fällung (11) lassen sich die $\beta$-Lipoproteine des Serums von den $\alpha$-Lipoproteinen trennen. Dazu werden $3 \mathrm{~m} l$ Serum bei $+4^{\circ}$ mit $0,15 \mathrm{ml}$ einer $1 \mathrm{M} \mathrm{MnCl}_{2}^{-}$ Lösung, die $6 \mathrm{mg}$ Heparin- $\mathrm{Na}$ enthält, vermischt und die $\beta$-Lipoproteine ausgefällt. Nach $15 \mathrm{Min}$. trennt man Überstand und Niederschlag durch übliches Zentrifugieren. Der Niederschlag wird mit 0,9proz. $\mathrm{NaCl}$ Lösung auf $3 \mathrm{~m} l$ aufgefüllt. Überstand und Niederschlag

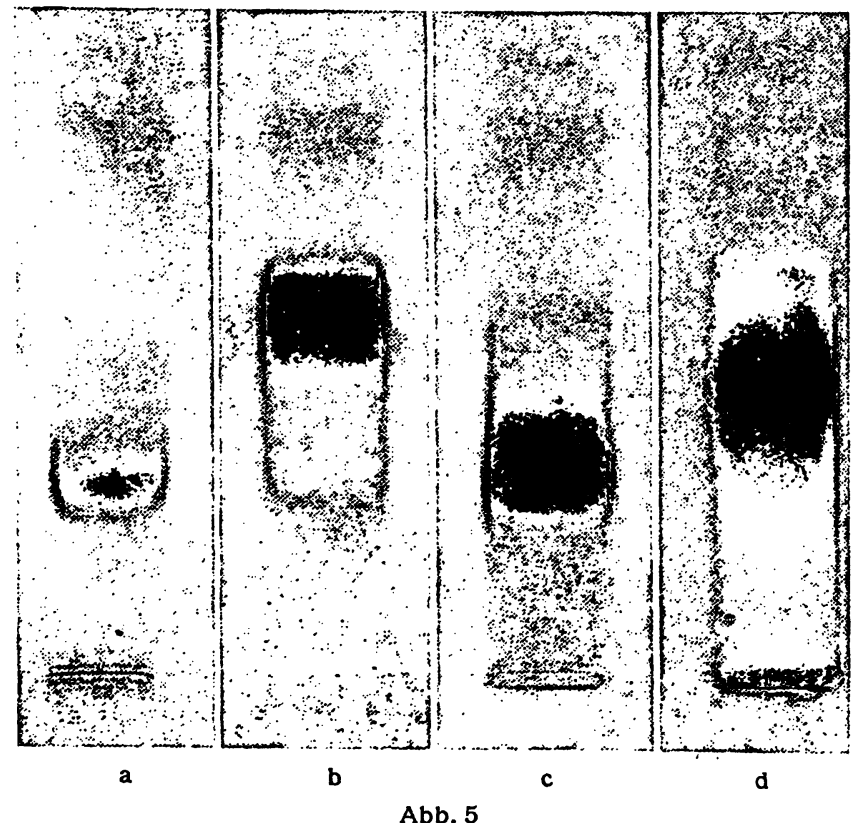

Lipoproteinelektrophorese (Standardmethode) eines normalen Serums (a), eines Serums mit prä- $\beta$-Lipoproteinen (b), einer familiären Hyper-
cholesterinämie (c) und einer Hyperlipämie ungeklärter Genese (d) werden wie das Serum (siehe Methodik) in die Lipoproteinelektrophorese eingesetzt (Abb. 4).

\section{Anvendungsbeispiele}

Mit der beschriebenen Modifikation der Lipoproteinelektrophorese in Agarosegel (0,8\%) mit Albuminzusatz $(0,26 \%)$ lassen sich die bisher für die Klassifizierung der Hyperlipidämien als wichtig erkannten Lipoproteinfraktionen: Chylomikronen, $\beta$-Lipoproteine und prä- $\beta$ Lipoproteine mit einer für die densitometrische Auswertung wesentlichen homogenen Substanzverteilung innerhalb der Banden klar voneinander trennen. Die $\alpha=$ Lipoproteine bilden regelmäßig zwei Fraktionen, deren Größe wechseln kann. Neben diesen bekannten Fraktionen findet man im Bereich der $\beta$ - und prä- $\beta$-Fraktion bei einzelnen Patienten mit einer Hyperlipämie zusätzliche Lipoproteinbanden, auf die auch RAPP und Kahlke (6) sowie Noble (8) hingewiesen haben. In Abbildung 5 sind physiologische und pathologische Lipoproteinverteilungsmuster dargestellt.

\section{Diskussion}

Gegenüber dem Papier begünstigt die Agarose durch schärfere Banden eine bessere Trennung und gewährleistet eine klare, durchsichtige Schicht. Diese Eigenschaft macht Agarosegel für densitometrische Auswertungen sehr geeignet, da eine gleichmäßig niedrige Leerabsorption der Schicht einen ruhigen Kurvenverlauf und ein enger Lichtspalt eine klare Abgrenzung der Banden erlaubt. Weitere Möglichkeiten bieten sich durch die Anwendung als präparative Gelelektrophorese und die Kombination mit dem immunologischen Nachweis bestimmter Proteine im gleichen Trägermedium. Diese Vorteile der Agarose wiegen den größeren Aufwand bei der Herstellung der Schicht sowie beim Fixieren und Trocknen des Gels auf. Zur gleichen Beurteilung der Agarose als Träger bei der Lipoproteinelektrophorese kamen auch KaHLKE und SCḦLIERF (12) sowie Noble (8).

RApp und KahLKe (6) trennten die Serumlipoproteine an reinen Agarosegelschichten ( $0,8 \%$ Agarose). Es gelang eine wesentlich bessere Trennung $\operatorname{der} \beta$-von den prä- $\beta$-Lipoproteinen als dies auf Papier möglich ist, aber die Form der Banden, vor allem $\operatorname{der} \beta$-Lipoproteine, war unsymmetrisch mit einer Bandenschärfung in Richtung auf den Auftragsort und sichelförmiger Verformung (Abb. 2). Gleichzeitig überschreitet die kathodische Anhäufung der $\beta$-Lipoproteine bald die Grenze der Proportionalität zwischen Lipidmenge und Farbintensität.

Noble (1968) prüfte die Beziehung zwischen der Agatosekonzentration in der Schicht und der Wanderung der Fraktionen und fand, daß die Trennung von prä- $\beta$ - und $\beta$-Lipoproteinen sich mit abnehmender Konzentration von Agarose verbesserte. $\mathrm{Er}$ benützte deshalb eine Schicht aus 0,5 proz. Agarose, die durch $0,6 \%$ Agar gehärtet wird, und einen Zusatz von 0,5\% Albumin. Diese Schicht ist aber sehr weich und leicht verletzlich. Nach unseren Erfahrungen ist die Ver- 
besserung der Trennung nicht entscheidend, und eine Schicht mit 0,8proz. Agarose vorzuziehen, da sie so fest ist; daß man für präparative $Z$ wecke die feuchte Schicht schneiden und von einer Platte zur anderen übertragen kann. Die von Nobre vorgeschlagenen Filmstreifen als Träger der Schicht bieten gegenüber dünnen Glasplatten keinen Vorteil, da diese auch gut lichtdurchlässig und stabiler sind.

Aufgrund der Erfahrungen mit der Lipoproteinelektrophorese auf Papier (2) war zu erwarten, daß auch ein Zusatz von Albumin zur Agarose eine günstigere Bandenform bringt (7). Noble (8) erzielte durch einen Albuminzusatz von $0,5 \%$ zu Agarose- und Agarose-AgarSchichten homogene Banden als Voraussetzung für eine gute Densitometrie. Unserer Erfahrung nach ist der Albuminzusatz zum Agarosegel schon in einer Konzentration von $0,05 \%$ voll wirksam und bringt bei höheren Konzentrationen keinen weiteren Vorteil. Gegenüber dem Verfahren von NoBLe hat unsere Modifikation, möglicherweise durch Wegfall von Agar, den Vorzug, regelmäßig zwei $\alpha$-Lipoproteinbanden nachzuweisen (16). Die Einteilung der Hyperlipoproteinämien durch FREDRICKSON (13) brachte für die Erforschung der Hyperlipidämien einen entscheidenden Fortschritt. Nach Vergleich der Elektrophorese an Papier und Agarose mit der Ultrazentrifuge durch FreDRICKSON und Mitarbeiter (14) und Noble und Mitarbeiter (15) muß man jedoch im Bereich $\operatorname{der} \beta$ - und prä- $\beta$-Lipoproteine weitere Fraktionen vermuten, deren Abklärung bisher dem Speziallaboratorium mit der Ultrażentrifuge vorbehalten blieb. Verbesserungen der elektrophoretischen Trennung, wie sie mit der Anwendung des Agarosegels mit Albuminzusatz gezeigt wurden, erweitern die Möglichkeiten für klinisch-chemische Untersuchungen an einem großen Patientengut.

\section{Literatur}

1. Maurer, W., Klin. Wschr. 30, 323 (1952). - 2. Lees, R. S. und F. T. Hatch, J. Laborat. Clin. Med. S. Louis 61, 518 (1963). 3. Fredrickson, D. S. und R. S. LeEs, Circulation 31, 321 (1965). 4. Fredrickson, D. S. und R. S. LeEs, The Metabolic Basis of Inherited Disease, J. B. Stanbury, J. B. WYNGaARden, D. S. FredrICKSon, 2nd Edition, McGraw-Hill Book Company New York (1966). - 5. KaHLKE, W. und W. Rapp, Verh. Dtsch. Ges. inn. Med. 73, 828 (1967). - 6. RAPP, W. und W. KaHLKE, Clin. chim. Acta Amsterdam 19, 493 (1968). - 7. Harch, F. T. und R. S. Lees: Adv. Lipid Res., Vol. 6, Academic Press, New YorkLondon (1968). - 8. Noble, R. P., J. Lipid Res. 9, 693 (1968). 9. Grabar, P. und P. Purdin, Immunelektrophoretische Analyse.
Elsevier Publ. Comp. Amsterdam (1964). - 10. Zöllner, N. und D. EBERHAGEN, Untersuchung und Bestimmung der Lipoide im Blut, S. 122, Springer-Verlag, Berlin-Heidelberg-New York (1965). 11. Burstein, M. und J. Samaille, Clin. chim. Acta Amsterdam 5, 609 (1960). - 12. KahLKe, W. und G. SchlierF, Klin. Wschr. 6, 330 (1968). - 13. FredrICKSON, D. S., R. I. LeVY und R. S. LeEs, New Engl. J. Med. 276, 32 (1967). - 14. Fredrickson, D. S., R. J. Levy und F. T. Lindgren, J. Clin. Invest. 47, 11 (1968). - 15. Noble, R. P., F. T. Hatch, J. A. Mazrimas, F. T. Lindgren, L. C. Jensen und G. L. Adamson, Lipids 4, 55 (1969). 16. LEVY, R. T. und D. S. FredRICKson, J. clin. Invest. 44, 426 (1965).
Prof. Dr. N. Zöllner 8000 München 15 Pettenkoferstr. 8 a 